\section{A Mean Level Adaptive Detector Using Nonconcurrent} Data

KARL GERLACH, Member, IEEE

Naval Research Laboratory

\section{INTRODUCTION}

The matched filter detector (MFD) is a common implementation used in deciding whether a desired signal is present or not. The MFD is illustrated in Fig. 1. The output of $N$ sensors are input to the MFD. If the covariance matrix, $R_{x x}$, of the inputs, $x_{1}, x_{2}, \ldots, x_{N}$, is known a priori and the desired signal can be represented by an $N$-length vector $s$, then the matched filter weights, $a_{1}, a_{2}, \ldots, a_{N}$ are given by $\mathbf{a}=R_{x x}^{-1} \mathrm{~s}$ where $\mathbf{a}=\left(a_{1}, a_{2}, \ldots, a_{N}\right)^{\mathrm{T}}$ and $\mathrm{T}$ denotes transpose [1]. The output of the matched filter is $\mathbf{a}^{\mathrm{H}} \mathbf{x}$ where $\mathbf{x}=\left(x_{1}, x_{2}, \ldots, x_{N}\right)^{\mathrm{T}}$ and $\mathrm{H}$ denotes conjugate transpose. This output is square law detected and compared against a threshold. A detection is declared if this threshold is exceeded.

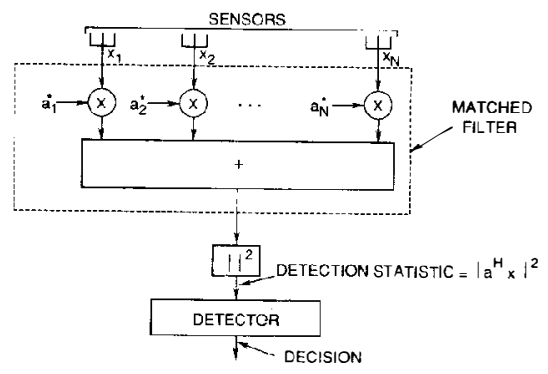

Fig. 1. Matched filter detector.

For known covariance matrix, threshold, and signal-to-noise power ratio, the detection probability $P_{D}$, and false alarm probability $P_{F}$, have been derived [1]. In some applications the covariance matrix is not known a priori and is estimated. The matched filter weighting is then determined by using what has been termed, the sample matrix inversion (SMI) algorithm [2]. Detection results for the output noise powcr residue of an adaptive matched filter which uses the SMI are given in [2-4].

Kelly [5] derived an adaptive detector under the Gaussian assumption, using the maximum likelihood (ML) estimator for the unknown parameters of the maximum likelihood ratio test, i.e., the unknown covariance matrix and the unknown signal amplitude. This detection scheme is known as the generalized likelihood ratio test [1]. The amplitude of the desired signal was assumed nonrandom. Input data consisted of a primary data vector of length $N$, which might contain the desired signal and a number of secondary data vectors which do not contain the desired signal. $R_{x x}$ is estimated from these secondary data vectors using the SMI algorithm. Performance results for $P_{D}$ and $P_{F}$ were presented in [5]. Expressions for $P_{D}$ and $P_{F}$ were derived that are a function of the number of statistically independent secondary data vectors, the number of input channels $N$, the detector threshold, and the input signal-to-noise power ratio. It was noted

$\overline{0018-9251 / 94 \$ 4.00 ~(C) ~} 1994$ IEEE 
that $P_{F}$ did not depend on $R_{x x}$ (a statistical measure of the external noise environment). Hence this detector exhibited the desirable constant false alarm rate (CFAR) property of having the $P_{F}$ bc independent of the covariance matrix. Additional research in the area is contained in $[6,7]$.

We consider a different form of CFAR adaptive detection whereby a mean level detector (MLD) is employed $[8,9]$. For this detcction scheme as in Kelly's [5], a fixed-number of secondary data vectors which do not contain the desired signal are used to estimate $R_{x x}$. A number of primary data vectors are processed through the matched filter and square law detected. Thereafter, one of the resultant outputs is selected as a candidate for detection and the rest of the resultant outputs powers are averaged and multiplied by an arbitrary number to form the threshold. Also in this work, a random desired signal is treated. In particular, results for the Rayleigh target model are presented. Formulas for $P_{D}$ and $P_{F}$ are derived for what we term the mean level adaptive detector (MLAD) and again it is shown that this detector exhibits the CFAR property of the $P_{F}$ being independent of the input covariance matrix.

The pertinant assumptions for this analysis are the following.

1) Input noises are complex zero-mean stationary Gaussian random variables (RVs). The real and imaginary parts of a given input noise sample are independent and identically distributcd (IID). An $\mathrm{RV}$ with these characteristics is called a circular Gaussian process. If the mean is non-zero, we call this a noncentral Gaussian process.

2) Input noise samples are temporally statistically independent.

3) The secondary data is statistically independent of the primary data.

4) The desired signal is present in the candidate primary data vector. It is not in the secondary data or the primary data vectors used to form the threshold.

We note that assumptions $1-4$ were also used in [5].

\section{MEAN LEVEL ADAPTIVE DETECTOR DESCRIPTION}

The MLAD is illustrated in Fig. 2. A batch or block of input data (called secondary input data) is used to calculate the adaptive weights. On each of the $N$ input channels, we measure $K$ temporally independent samples.

Define $X$ as the $N \times K$ matrix of secondary input data. The $n$th row represents the $K$ temporally independent samples on the $n$th channel. The samples in the $k$ th column are assumed to be time coincident. The variable $\mathbf{s}$ is the desired steering vector of length $N$, and $\hat{R}_{x x}$ is the $N \times N$ estimated input covariance matrix.

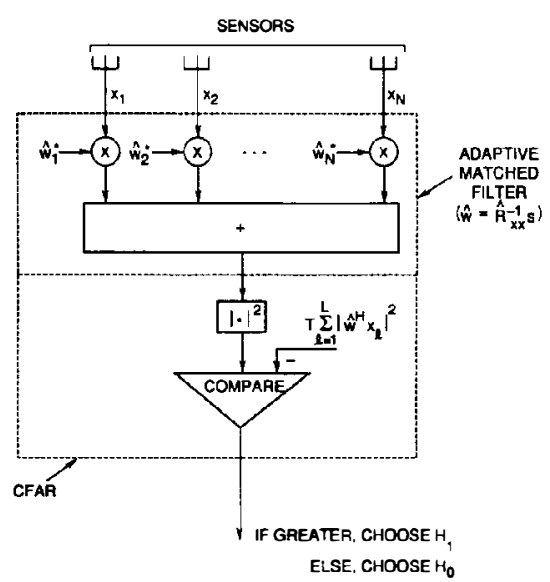

Fig. 2. Mean level adaptive detector.

The optimal estimate $\hat{w}$ of the optimal $N$-length weighting vector is given by [2]

$$
\hat{\mathbf{w}}=\hat{R}_{x x}^{-1} \mathbf{s}
$$

where

$$
\hat{R}_{x x}=\frac{1}{K} X X^{\mathrm{H}} .
$$

Equations (1) and (2) are essentially the SMI algorithm for computing the matched filter or Weiner weights.

This optimal estimate is then applicd to another temporally independent set of data called the primary input data. The primary input vectors are of length $N$ and their elements are assumed temporally independent. Let $\mathbf{x}$ be the candidate primary input data vector of length $N, \mathbf{x}_{l}$ be the $l$ th primary input data vector of length $N$, used to compute the detector threshold, $l=1,2, \ldots, L, L$ be the number of primary input data vectors used to compute the detector threshold, and $T$ be the MLD threshold constant.

The MLAD rule is given mathematically as

$$
\left|\hat{\mathbf{w}}^{\mathrm{H}} \mathbf{x}\right|^{2} \underset{\mathrm{H}_{0}}{\stackrel{\mathrm{H}_{1}}{\gtrless}} T \sum_{l=1}^{L}\left|\hat{\mathbf{w}}^{\mathrm{H}} \mathbf{x}_{l}\right|^{2}
$$

wherc $|\cdot|$ denotes magnitude, $\mathrm{H}_{0}$ is the hypothesis that no desired signal is present, and $\mathrm{H}_{1}$ is the hypothesis that a desired signal is present. We note in the form of this detector, the standard CFAR procedure is to normalize the candidate primary test statistic by the average of the estimated power of the other primary test statistics (note, $1 / L$ has been incorporated into $T$ ). The probabilities of false alarm and detection probabilities are defined as

$$
\begin{aligned}
& P_{F}=\operatorname{Pr}\left\{\left|\hat{\mathbf{w}}^{\mathrm{H}} \mathbf{x}\right|^{2}>T \sum_{l=1}^{L}\left|\hat{\mathbf{w}}^{\mathrm{H}} \mathbf{x}_{l}\right|^{2} \mid \mathrm{H}_{0}\right\} \\
& P_{D}=\operatorname{Pr}\left\{\left|\hat{\mathbf{w}}^{\mathrm{H}} \mathbf{x}\right|^{2}>T \sum_{l=1}^{L}\left|\hat{\mathbf{w}}^{\mathrm{H}} \mathbf{x}_{l}\right|^{2} \mid \mathrm{H}_{1}\right\} .
\end{aligned}
$$


We now introduce a matrix transform on the input channels which does not change $P_{D}$ or $P_{F}$, but greatly simplifies the analysis. Let $R_{x x}$ be the $N \times N$ covariance matrix of the input channels. Assume that the matrix is nonsingular. There exist [2] an $N \times N$ matrix $A$, which 1 ) spatially whitens the $N$ input channels, 2) normalizes each input channel to have noisc power equal to one, and 3) places all of the signal energy in the first channel such that the transformed signal vector $\tilde{\mathbf{s}}$ is given by

$$
\tilde{\mathbf{s}}=A \mathbf{s}=(u, 0,0, \ldots, 0)^{\mathrm{T}}
$$

where $u$ represents the transformed desired signal voltage.

Define $Z=A X$ as the $N \times K$ matrix of transformed input data. Each sample is temporally and spatially independent with variance equal to 1 . Also, define $\mathbf{z}_{l}=A \mathbf{x}_{l}$ as the $l$ th transformed MLD primary data vector, $l=1,2, \ldots, L$, and $\mathrm{z}=A \mathbf{x}$ as the candidate primary input data vector.

It is straightforward to show that the weight $\mathbf{w}$ of the transformed data is given by

$$
\mathbf{w}=\left(A^{\mathrm{H}}\right)^{-1} \hat{\mathbf{w}} .
$$

The transformed MLAD rule is illustrated in Fig. 3 and is given by

$$
\left|\mathbf{w}^{\mathrm{H}_{2}}\right|^{2} \underset{\mathrm{H}_{0}}{\stackrel{\mathrm{H}_{1}}{\gtrless}} T \sum_{l=1}^{L}\left|\mathbf{w}^{\mathrm{H}} \mathbf{z}_{\ell}\right|^{2} .
$$

By substituting for $\mathbf{w}, \mathbf{z}$, and $\mathbf{z}_{l}$ with $\left(A^{\mathrm{H}}\right)^{-1} \hat{\mathbf{w}}, A \mathbf{x}$, and $A \mathbf{x}_{l}$, respectively, we find that (3) results. Hence, the equivalence of the two decision rules is proven. Thus

$$
\begin{aligned}
& P_{F}=\operatorname{Pr}\left\{\left|\mathbf{w}^{\mathrm{H}} \mathbf{z}\right|^{2}>T \sum_{l=1}^{L}\left|\mathbf{w}^{\mathrm{H}} \mathbf{z}_{l}\right|^{2} \mid \mathrm{H}_{0}\right\} \\
& P_{D}=\operatorname{Pr}\left\{\left|\mathbf{w}^{\mathrm{H}} \mathbf{z}\right|^{2}>T \sum_{l=1}^{L}\left|\mathbf{w}^{\mathrm{H}} \mathbf{z}_{l}\right|^{2} \mid \mathrm{H}_{1}\right\} .
\end{aligned}
$$

We see from (8) that an arbitrary scaling factor multiplying both sides of the decision rule does not change the rule. Henceforth, we set the first element of w equal to 1 and define

$$
\mathbf{w}=\left(1, w_{2}, w_{3}, \ldots, w_{N}\right)^{\mathrm{T}} .
$$

Finally we note that as $K \rightarrow \infty$, then $w_{n} \rightarrow 0, n=$ $2,3, \ldots, N$. This is because the Weiner weights are effectively achieved after the transformation by $A$ [4]. Hence the adaptive weights $(n=2,3, \ldots, N)$, computed after this transformation are perturbations about their optimal values which are zero.

\section{PROBABILITY OF FAISE ALARM}

In this section, we derive $P_{F}$ and show that the adaptive detection scheme discussed in Section II does

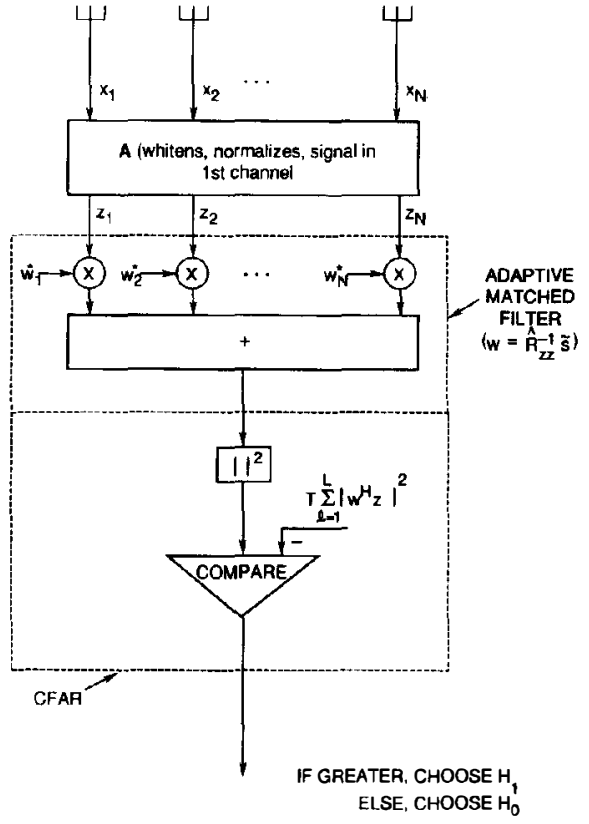

Fig. 3. Transformed MLAD.

indeed exemplify the qualities of a CFAR processor, i.e., the $P_{F}$ is independent of the external noise environment. To this end define $P_{F}(w)$ equal to probability of false alarm conditioned on knowing $\mathbf{w}$,

$$
\begin{aligned}
\nu & =\frac{\mathbf{w}^{\mathrm{H}} \mathbf{z}}{\|\mathbf{w}\|}, \\
\nu_{l} & =\frac{\mathbf{w}^{\mathrm{H}} \mathbf{z}_{l}}{\|\mathbf{w}\|}, \quad l=1,2, \ldots, L
\end{aligned}
$$

where $\|\mathbf{w}\|=\sqrt{\mathbf{w}^{\mathrm{H}_{\mathbf{w}}}}$. For $\|\mathbf{w}\|>0$ and under $\mathrm{H}_{0}$ it is straightforward to show that $\nu$ and $\nu_{l}, l=1,2, \ldots, L$, are independent identically distributed (IID) circular Gaussian processes with zero mean and variance equal to one. The decision rule given by (8) can be rewritten as

$$
|\nu|^{2} \underset{\mathrm{H}_{0}}{\stackrel{\mathrm{H}_{1}}{\gtrless}} T \sum_{l=1}^{L}\left|\nu_{l}\right|^{2} .
$$

For the above decision rule it is well-known [8] that

$$
\operatorname{Pr}\left\{|\nu|^{2}>T \sum_{l=1}^{L}\left|\nu_{l}\right|^{2}\right\}=\frac{1}{(1+T)^{L}}
$$

which is a special case of the $F$-distribution. Thus

$$
P_{F}(\mathbf{w})=\frac{1}{(1+T)^{L}}
$$

which does not depend on w. Hence

$$
P_{F}=\frac{1}{(1+T)^{L}} .
$$

We notc that $P_{F}$ is a function only of the arbitrary threshold $T$ and $L$, the number of MLD samples. 


\section{DETECTION PROBABILITY}

In this section, we derive the detection probability $P_{D}$ associated with the MLAD given a general probability density function (pdf) of the desired signal amplitude. We assume the desired signal phasc is uniformly distributed on $[0,2 \pi)$. Under $\mathrm{H}_{1}$

$$
\mathbf{z}=\left(u+n_{1}, n_{2}, n_{3}, \ldots, n_{N}\right)^{\mathrm{T}}
$$

where $\mathbf{n}=\left(n_{1}, n_{2}, \ldots, n_{N}\right)^{\mathrm{T}}$ is an additive Gaussian noise vector with zero mean and element variancc equal to one and $u$ is the desired signal voltage through the matrix transform $A$. If $a$ is the desired signal voltage before the $A$ transformation, then $u=a\left(\mathbf{s}^{\mathrm{H}} R_{x x}^{-1} \mathrm{~s}\right)^{1 / 2}$. Under $\mathrm{H}_{1}$, we adopt the notation

$$
\begin{aligned}
P_{D}(\bullet)= & \text { probability of detection conditioned } \\
& \text { on the arguments, } \bullet
\end{aligned}
$$

Notc we are assuming that the desired signal is not present in the primary data vectors used to form the detector threshold. Define $\nu_{l}, l=1,2, \ldots, L$ as done by (13). Also set

$$
\begin{aligned}
\nu & =\frac{\mathbf{w}^{\mathrm{H}_{\mathbf{z}}}}{\|\mathbf{w}\|}=\frac{\mathbf{w}^{\mathrm{H}_{\tilde{\mathbf{s}}}}}{\|\mathbf{w}\|}+\frac{\mathbf{w}^{\mathrm{H}} \mathbf{n}}{\|\mathbf{w}\|} \\
& =\frac{u}{\|\mathbf{w}\|}+\frac{\mathbf{w}^{\mathrm{H}} \mathbf{n}}{\|\mathbf{w}\|} .
\end{aligned}
$$

We set

$$
u_{0}=\frac{u}{\|\mathbf{w}\|}
$$

and

$$
\nu^{\prime}=\frac{\mathbf{w}^{\mathrm{H}} \mathbf{n}}{\|\mathbf{w}\|} .
$$

Now $\nu$ is a noncentral Gaussian process with a mean equal to $u_{0}$ and variance equal to one. As before, $\nu_{l}$ are IID circular Gaussian processes with zero mean and variance equal to one. They are also independent of $\nu$.

The decision rule given by (8) can be rewritten as

$$
\left|u_{0}+\nu^{\prime}\right|^{2} \underset{\mathrm{H}_{0}}{\stackrel{\mathrm{H}_{1}}{\gtrless}} T \sum_{l=1}^{L}\left|\nu_{l}\right|^{2} .
$$

Set

$$
\begin{aligned}
& r=\left|u_{0}+\nu^{\prime}\right|^{2} \\
& \eta=T \sum_{l=1}^{L}\left|\nu_{l}\right|^{2} .
\end{aligned}
$$

If the desired signal phase of $a$ is uniformly distributed on $[0,2 \pi)$ then the phase of $u$ is the same. Set $\lambda=|u|$ and $\lambda_{0}=\left|u_{0}\right|$. Under the IID Gaussian assumption, it can be shown [1] that the pdf of $r$, denoted by $p_{r}(r)$ is given by

$$
p_{r}(r)=e^{-\left(r+\lambda_{0}^{2}\right)} I_{0}\left(2 \lambda_{0} \sqrt{r}\right)
$$

where $I_{0}$ is the modified 0th-order Bessel function of the first kind. The distribution of $\eta$ is the chi-square distribution with pdf given by

$$
\rho_{\eta}(\eta)=\frac{1}{T^{L}}\left(\frac{1}{L-1) !} \eta^{L-1} e^{-\eta / T} .\right.
$$

This last result was obtained under conditioning by $\mathbf{w}$, but as we see, is independent of $\mathbf{w}$.

Now

$$
\operatorname{Pr}\{r>\eta\}=\int_{0}^{\infty} \int_{\eta}^{\infty} p_{r}(r) p_{\eta}(\eta) d r d \eta
$$

and

$$
P_{D}(\mathbf{w}, u)=P_{D}\left(u_{0}\right)=\operatorname{Pr}\{r>\eta\} .
$$

Again, the last quantity in this equation is obtained under conditioning by $\mathbf{w}$, but is independent of $\mathbf{w}$. Define

$$
\begin{aligned}
& q_{0}=\frac{1}{\|\mathbf{w}\|^{2}} \\
& u_{0}=u \sqrt{q_{0}} .
\end{aligned}
$$

Brennan and Rccd [2] showed that $q_{0}$ has the beta pdf which is given by

$$
p_{q_{\bullet}}\left(q_{0}\right)=\frac{K !}{(N-2) !(K-N+1) !}\left(1-q_{0}\right)^{N-2} q_{0}^{K-N+1},
$$

$$
0 \leq q_{0} \leq 1
$$

Set $q=\sqrt{q_{0}}$. It is straightforward using elementary probability theory to show that

$$
\begin{array}{r}
p_{q}(q)=\frac{K !}{2(N-2) !(K-N+1) !}\left(1-q^{2}\right)^{N-2} q^{2(K-N)+1,} \\
0 \leq q \leq 1 .
\end{array}
$$

Thus the joint pdf of $\lambda$ and $q$ which are assumed to be independent RVs is given by

$$
P_{\lambda, q}(\lambda, q)=p_{\lambda}(\lambda) p_{q}(q) .
$$

At this point, we note that

$$
P_{D}(\mathbf{w}, \lambda)=P_{D}\left(\lambda_{0}\right)=P_{D}(\lambda, q) .
$$

Thus knowing the pdf of $\lambda_{0}$ is not necessary since we have the joint pdf of $p_{\lambda, q}(\lambda, q)$.

Finally the detection probability is found as

$$
P_{D}=\int_{0}^{\infty} \int_{0}^{1} P_{D}(\lambda, q) p_{\lambda, q}(\lambda, q) d q d \lambda
$$


or

$$
\begin{aligned}
P_{D}= & c_{0} \int_{0}^{\infty} \int_{0}^{1} \int_{0}^{\infty} \int_{y}^{\infty} e^{-\left(r+\left(\lambda^{2} / q^{2}\right)\right)} I_{0}\left(2 \frac{\lambda}{q} \sqrt{r}\right) \\
& \cdot y^{L-l} e^{-y / T}\left(1-q^{2}\right)^{N-2} q^{2(K-N)+1} \\
& \times p_{\lambda}(\lambda) d r d y d q d \lambda
\end{aligned}
$$

wherc

$$
c_{0}=\frac{K !}{2(N-2) !(K-N+1) !} \frac{1}{T^{L}(L-1) !} .
$$

We note that the signal parameters are contained in the general pdf, $p_{\lambda}(\lambda)$, which can be easily derived from the pdf of $|a|$ since $\lambda=|a|\left(\mathbf{s}^{\mathrm{H}} R_{x x}^{-1} \mathbf{s}\right)^{1 / 2}$. Since the output noise power residue of the matched filter $(K=\infty)$ is normalized to 1 , the quantity $\lambda^{2}$ is actually the output signal-to-noise ratio of the matched filter for a constant input signal amplitude.

\section{DETECTION PROBABILITY: RAYLEIGH SIGNAL}

In this section we derive an expression for $P_{D}$ for the special case when the envelope of desired input signal $u$ is Rayleigh distributed. We define $\nu_{l}$ as done by (13) and $\nu$ as done by (19). Implicit in the assumption that $|u|$ is Rayleigh distributed is that $u$ is a circular Gaussian process with zero mean and variance equal to $\sigma_{u}^{2}$. Thus $\nu$ is circular Gaussian with zero mean and

$$
\sigma_{\nu}^{2}=\frac{\sigma_{u}^{2}}{\|\mathbf{w}\|^{2}}+1
$$

We repeat (14) for convenience:

$$
|\nu|^{2} \underset{\mathrm{H}_{0}}{\stackrel{\mathrm{H}_{1}}{\gtrless}} T \sum_{l=1}^{L}\left|\nu_{l}\right|^{2} .
$$

We define $\eta$ as in (24) and set

$$
r=|\nu|^{2} \text {. }
$$

The pdf of $r$ is given by

$$
p_{r}(r)=\frac{1}{\sigma_{\nu}^{2}} e^{-r / \sigma_{\nu}^{2}}
$$

Steenson [8] shows that for the assumed pdf that

$$
P_{D}(\mathbf{w})=\operatorname{Pr}\{r>\eta\}=\left[1+\frac{T}{\sigma_{\nu}^{2}}\right]^{-L} .
$$

Again set $q_{0}=1 /\|\mathbf{w}\|^{2}$. Thus (41) becomes

$$
P_{D}(\mathbf{w})=P_{D}\left(q_{0}\right)=\left[1+\frac{T}{\sigma_{u}^{2}} \overline{q_{0}+1}\right]^{-L} .
$$

We define $(S / N)_{\text {opt }}=\sigma_{u}^{2}=$ optimal signal-to-noise power ratio of the test statistic $z_{1}$. We note that $(S / N)_{\text {opt }}$ is also the optimal signal-to-noise power ratio of thc output of the matched filter where the optimal linear weight is given by $w=R_{x x}^{-1} \mathrm{~s}$. The pdf of $q_{0}$ is given by (30). It follows that

$$
\begin{aligned}
P_{D}= & \frac{K !}{(N-2) !(K-N+1) !} \\
& \times \int_{0}^{1}\left[1+\frac{T}{q_{0}\left(\frac{S}{N}\right)_{\mathrm{opt}}+1}\right]^{-L} \\
& \times\left(1-q_{0}\right)^{N-2} q_{0}^{K-N+1} d q_{0} .
\end{aligned}
$$

Using (17),

$$
T=P_{F}^{-1 / L}-1 .
$$

Substituting this expression into (43) results in

$$
P_{D}=c \int_{0}^{1}\left[\frac{q_{0}+a}{q_{0}+b}\right]^{L}\left(1-q_{0}\right)^{N-2} q_{0}^{K-N+1} d q_{0}
$$

wherc

$$
\begin{aligned}
& a=\left(\frac{S}{N}\right)_{\mathrm{opt}}^{-1} \\
& b=\left(\frac{S}{N}\right)_{\mathrm{opt}}^{-1} P_{F}^{-1 / L}
\end{aligned}
$$

and

$$
c=\frac{K !}{(N-2) !(K-N+1) !} .
$$

Wc note that as $K \rightarrow \infty$, then $q_{0} \rightarrow 1$. Thus the quiescent $P_{D}$ denoted by $P_{D}^{(q)}$ is given by

$$
P_{D}^{(q)}=\left[1+\frac{T}{\frac{\sigma_{u}^{2}}{\sigma_{\min }^{2}}+1}\right]^{-L} .
$$

Using (44) and $(S / N)_{\mathrm{opt}}=\sigma_{u}^{2} / \sigma_{\min }^{2}$, then

$$
P_{D}^{(q)}=\left[1+\frac{P_{F}^{-1 / L}-1}{\left(\frac{S}{N}\right)_{\mathrm{opt}}+1}\right]^{-L}
$$

\section{RESULTS}

In this section, we present some results on the detection probability $P_{D}$ of the MLAD versus the independent parameters: the probability of false alarm $P_{F}$; the steady state signal-to-noise output power ratio of the matched filter $(S / N)_{\text {opt }}$; the number of independent samples per channel $K$ used to calculate the sample covariance matrix; the order of the adaptive matched filter $N$; and thc number of samples $L$ used 


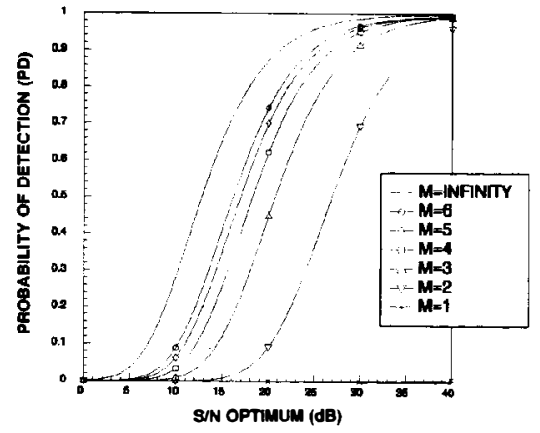

Fig. 4. $P_{D}$ vs. $(S / N)_{\text {opt }}, P_{F}=10^{-6}, N=2$.

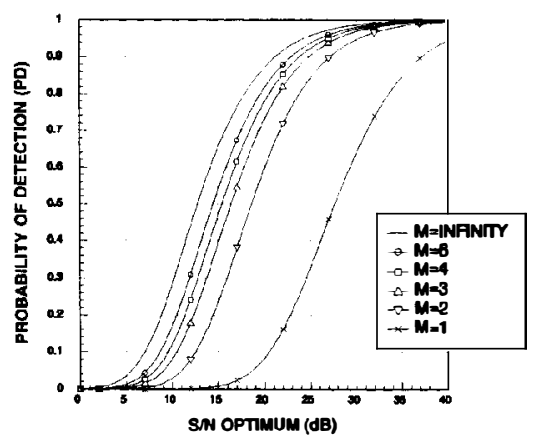

Fig. 5. $P_{D}$ vs. $(S / N)_{\text {opt }}, P_{F}=10^{-6}, N=5$.

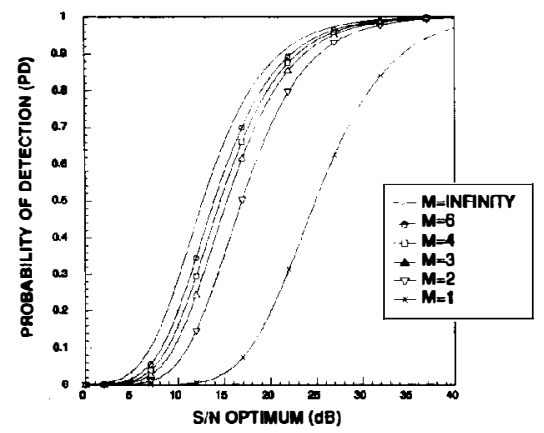

Fig. 6. $P_{D}$ vs. $(S / N)_{\text {opt }}, P_{F}=10^{-6}, N=10$.

to set the mean level threshold. The integral solution given by (45) was evaluated.

We set $K=M N$ where $M$ is a positive integer and use $M$ as an independent parameter called the degrees of freedom (DOF) factor. Plots of $P_{D}$ versus $(S / N)_{\text {opt }}$ and $M$ for $P_{F}=10^{-6}, 10^{-10}$ and various $N$ are shown in Figs. 4-13. We note that for these figures we have set $L=K-1$. This might be a logical choice for the number of samples used to set the threshold since all the samples except the candidate primary input data in a given batch of $K$ samples are used to set the threshold. Note that as $M \rightarrow \infty$, then $L \rightarrow \infty$, and that [1]

$$
P_{D}^{(q)}=P_{F}^{\left[1+(S / N)_{o p t}\right]^{-1}}
$$

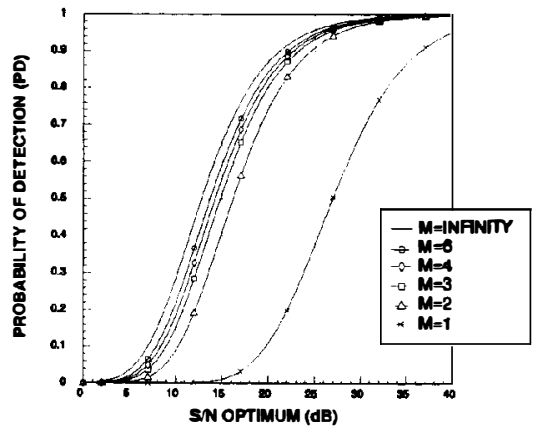

Fig. 7. $P_{D}$ vs. $(S / N)_{\text {opt }}, P_{F}=10^{-6}, N=30$.

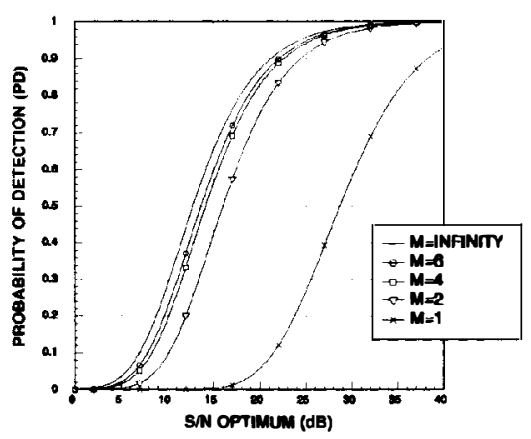

Fig. 8. $P_{D}$ vs. $(S / N)_{\text {opt }}, P_{F}=10^{-6}, N=50$.

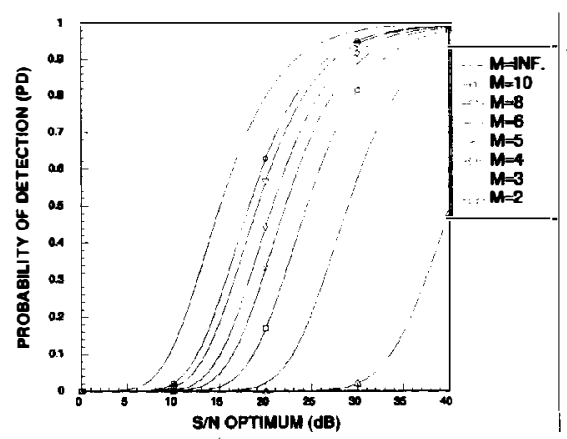

Fig. 9. $P_{D}$ vs. $(S / N)_{\mathrm{opt}}, P_{F}=10^{-10}, N=2$.

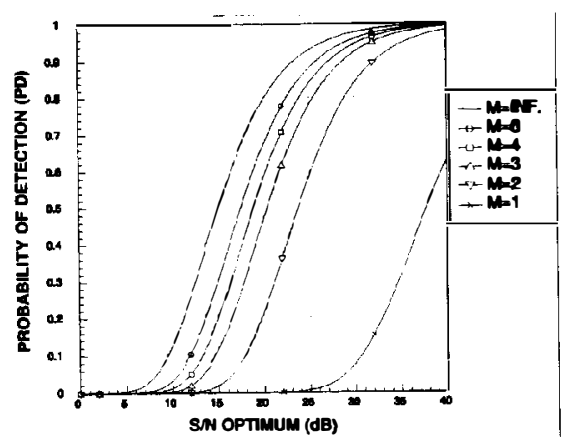

Fig. 10. $P_{D}$ vs. $(S / N)_{\text {opt }}, P_{F}=10^{-10}, N=5$. 


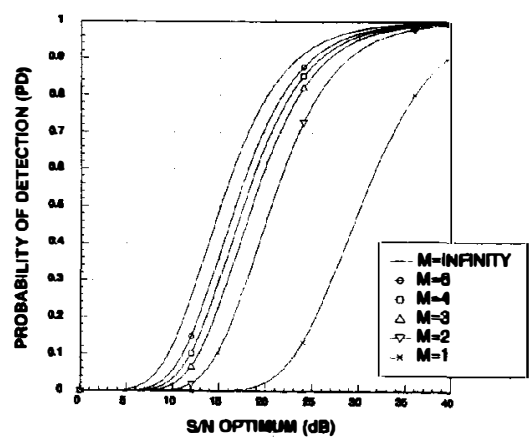

Fig. 11. $P_{D}$ vs. $(S / N)_{\text {opt }}, P_{F}=10^{-10}, N=10$.

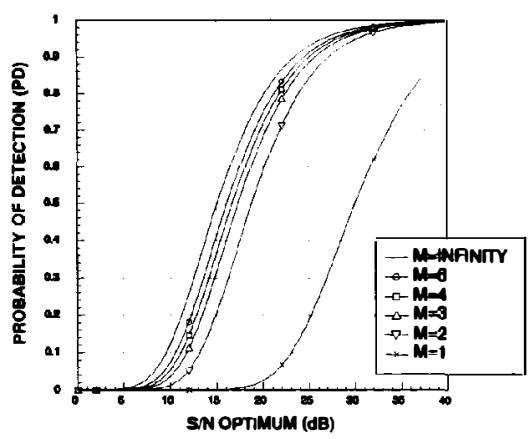

Fig. 12. $P_{D}$ vs. $(S / N)_{\mathrm{opt}}, P_{F}=10^{-10}, N=30$.

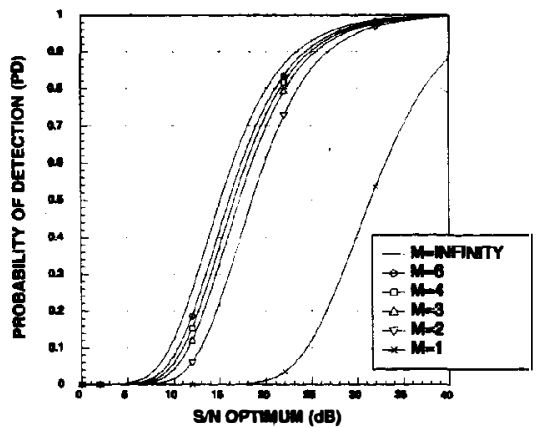

Fig. 13. $P_{D}$ vs. $(S / N)_{\text {opt }}, P_{F}=10^{-10}, N=50$

Kelly [5] defines the $(S / N)$ loss of an adaptive detector as the difference of required $(S / N)$ to obtain a given $P_{D}$ between a steady state $(M=\infty)$ detector and transient state ( $M$ finite) with all other independent parameters being equal. Define $M_{3} \mathrm{~dB}$ to be the DOF factor such that $(S / N)$ loss is nearest to but not exceeding $3 \mathrm{~dB}$. We make the following observations from Figs. 4-13.

1) The MLAD is slower to converge to its optimal $P_{D}(M=\infty)$ for lower ordered matched filters. For example, for most $P_{D^{5}}(0.1-0.9), P_{F}=10^{-6}$, if $N=2$ then $M_{3} \mathrm{~dB}=6$; if $N=10$ then $M_{3} \mathrm{~dB}=3$.

2) There is diminishing returns in convergence by using a larger DOF factor.

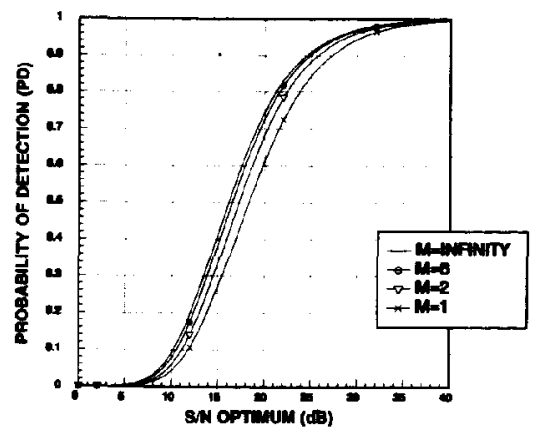

Fig. 14. $P_{D}$ vs. $(S / N)_{\text {opt }}, P_{F}=10^{-6}, N=2, L=10$.

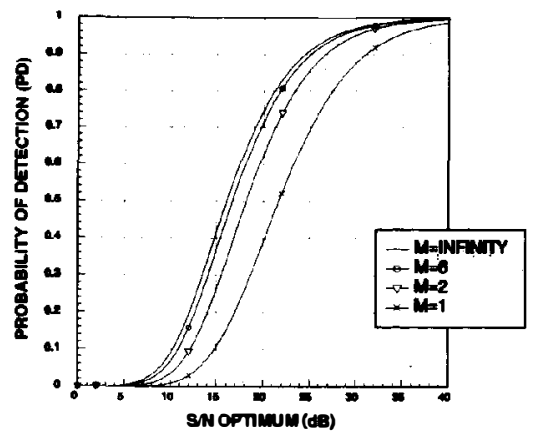

Fig. 15. $P_{D}$ vs. $(S / N)_{\text {opt, }} P_{F}=10^{-6}, N=5, L=10$.

3) Convergence slows for decreasing $P_{F}$. For example, for most $P_{D}(0.1-0.9)$ and $N=2$, if $P_{F}=$ $10^{-6}, M_{3} \mathrm{~dB}=6$, if $P_{F}=10^{-10}, M_{3} \mathrm{~dB}=10$.

We note that these trends were also observed by Kelly [5] for his adaptive detection algorithm.

Since in general the number of samples $L$ used to set the mean level detection threshold is arbitrary, we present two sets of curves, Figs. 14 and 15 where $L$ is not related to $K$. For these curves, $L=10$. Note that (50) and not (51) is used to evaluate $P_{D}^{(q)}$.

\section{SUMMARY}

Convergence results for an MLAD have been presented. The MLAD consists of an adaptive matched filter (for spatially correlated inputs) followed by an MLD. The optimal weights of the adaptive matched filter are estimated from one batch of data and applied to a statistically independent batch of nonconcurrent data. The threshold of the MLD is determined from the resultant data. Thereafter a candidate cell is compared against this threshold. Probabilities of false alarm and detection were derived as a function of the threshold factor, the order of the matched filter, the number of independent samples per channel used to calculate the adaptive matched filter weights $(K)$, the number of samples used to set the MLD threshold $(L)$, and the output signal-to-noise power 
ratio of the optimal matched filter. A number of performance curves were shown and discussed. It was shown for the particular case when, $L=K-1$, that the MLAD is slower to converge to its optimal value for lower ordered matched filters, there is diminishing returns in convergence performance when using more independent samples per channel, and convergence slows for increasing probability of false alarm.

\section{REFERENCES}

[1] Van Trees, H. L. (1968)

Detection, Estimation, and Modulation Theory, Vol. I. New York: Wiley, 1968.

[2] Reed, I. S., Mallett, J. D., and Brennan, L. E. (1974) Rapid convergence rate in adaptive arrays

IEEE Transactions on Aerospace and Electronic Systems, AES-10 (1974), 853-863.

[3] Gerlach, K., and Kretschmer, F. F, Jr. (1991)

Convergence properties of Gram-Schmidt and SMI adaptive algorithms, Part 2.

IEEE Transactions on Aerospace and Electronic Systems, 27, 1 (Jan. 1991), 83-91.

[4] Gerlach, K., and Kretschmer, F. F., Jr. (1990)

Convergence properties of Gram-Schmidt and SMI adapt ive algorithms.

IEEE Transactions on Aerospace and Electronic Systems, 26, 1 (Jan. 1990), 44-57.
[5] Kelly, E. J. (1986)

An adaptive detection algorithm.

IEEE Transactions on Aerospace and Electronic Systems, AES-22, 1 (Mar. 1986), 115-127.

[6] Nitzberg, R. (1984)

Detection loss of the sample matrix inversion technique. IEEE Transactions on Aerospace and Electronic Systems, AES-20, 6 (Nov. 1984), 824-827.

[7] Kelly, E. J. (1989)

Performance of an adaptive detection algorithm; rejection of unwanted signals.

IEEE Transactions on Aerospace and Electronic Systems, 25, 2 (Mar. 1989), 122-133.

[8] Steenson, B. O. (1968)

Detection performance of a mean-level threshold. IEEE Transactions on Aerospace and Electronic Systems, AES-4, 4 (July 1968), 529-534.

[9] Dillard, G. M. (1974)

Mean-level detection of nonfluctuating signals.

IEEE Transactions on Aerospace and Electronic Systems, AES-10 (Nov. 1974), 795-799.

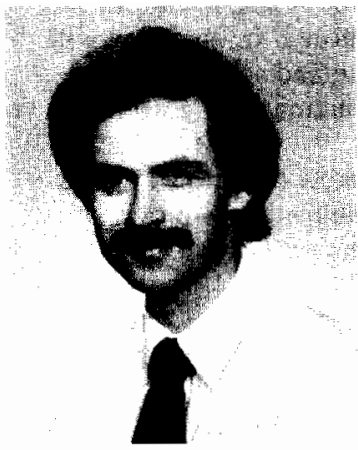

Karl Gerlach (M'81) was born in Chicago, IL. He received his B.S. in 1972 from the University of Illinois, Urbana, and his M.S. and D.Sc. from George Washington University, Washington, DC, in 1975 and 1981, respectively. All degrees are in electrical engineering.

Since 1972, he has been employed by the Naval Research Laboratory in Washington, DC. From 1972 to 1976, he worked on experimental submarine communications systems and from 1976 to the present he has been with the Radar Division where his research interests include adaptive signal processing and space-based radar.

Dr. Gcrlach was the 1986 recipient of the IEEE AESS Radar Systems Panel award. 